\title{
Neurophysiological correlates of error correction in sensorimotor-synchronization
}

\author{
P. Praamstra, ${ }^{\mathrm{a}, \mathrm{b}, *}$ M. Turgeon, ${ }^{\mathrm{a}}$ C.W. Hesse, ${ }^{\mathrm{a}}$ A.M. Wing, ${ }^{\mathrm{a}}$ and L. Perryer ${ }^{\mathrm{a}}$ \\ ${ }^{a}$ Behavioral Brain Sciences Centre, University of Birmingham, Birmingham, UK \\ ${ }^{\mathrm{b}}$ Department of Clinical Neurosciences, Queen Elizabeth Hospital, University of Birmingham, Birmingham, UK
}

Received 25 March 2003; revised 3 June 2003; accepted 3 June 2003

\begin{abstract}
In a sensorimotor synchronization task requiring subjects to tap in synchrony with an auditory stimulus, occasional perturbations (i.e., interval changes) in an otherwise isochronous sequence of auditory metronome stimuli are known to be compensated remarkably swift and with surprising precision, even when they are too small to be consciously perceived. To investigate the neural substrate and the informational basis of error correction in sensorimotor synchronization, we recorded movement-related, auditory-evoked, and error-related EEG potentials. Experiment 1 confirmed rapid adjustment to stimulus phase shifts, with faster correction of large (50 ms) compared to small (15 $\mathrm{ms}$ ) shifts. In addition to being corrected faster, there was overcorrection of the $50 \mathrm{~ms}$ shifts, attributed to engagement of period correction mechanisms. For $+50 \mathrm{~ms}$ shifts, a neural correlate of period correction was identified in the form of medial frontal cortex activation, preceded by an error-related brain potential (ERN). Auditory-evoked potential (AEP) amplitudes were sensitive to stimulus phase shifts of both large and small magnitude. Further experiments with a smaller magnitude $10 \mathrm{~ms}$ phase shift (Experiment 2) and passive auditory stimulation (Experiment 3) provided evidence that the modulation of AEP amplitudes is not due to metronome interval changes, but may represent auditory-somatosensory activation. Together, behavioral and neurophysiological data support the hypothesis that phase correction is a largely automatic process, not dependent on conscious perception of changes in timing. By contrast, perceivable phase shifts may invoke timekeeper adjustments accompanied by medial frontal cortex activity.
\end{abstract}

(C) 2003 Elsevier Inc. All rights reserved.

\section{Introduction}

Synchronizing a rhythmic movement, like tapping, to an auditory stimulus is a simple, undemanding task that nonetheless comprises key aspects of sensorimotor function. Tapping, both in synchronization and in continuation mode (i.e., after the pacing stimulus ceases), has been used extensively to study timing aspects of sensorimotor function. Formal/statistical modeling of such rhythmic motor behavior has helped to establish the notion of a central timekeeper (Wing and Kristofferson, 1973a, 1973b). In conjunction with neuroimaging (Harrington et al., 1998; Jäncke et al., 2000; Rao et al., 1997; Stephan et al., 2002) and neurosensing techniques (Fuchs et al., 2000; Mayville et al., 2001;

\footnotetext{
* Corresponding author. Behavioral Brain Sciences Centre, University of Birmingham, Birmingham B15 2TT, UK. Fax: +44-121-414-4897.

E-mail address: p.praamstra@bham.ac.uk (P. Praamstra).
}

Müller et al., 2000; Tecchio et al., 2000) tapping has recently been used to explore the neural basis of timing and the temporal control of action.

Maintaining synchrony between movements and pacing stimuli requires a monitoring and error-correction process that ensures optimal alignment of movements and stimulus sequence. To probe the workings of this process, Repp (2000, 2001a, 2001b) and others before him (e.g., Hary and Moore, 1985; Thaut et al, 1998a, 1998b) used perturbations of the temporal regularity of the pacing stimulus. Importantly, this research has shown that the correction of timing perturbations is not limited by the conscious detection threshold for changes in stimulus intervals or the perception threshold for changes in the synchronization error. Thus, for the temporal control of action, the motor system seems to have access to more accurate temporal information than is available for conscious auditory temporal judgment (Repp, 2000). 
Two-level timing models distinguish between a central level of timing control ("timekeeper") and a peripheral motor implementation level (Wing and Kristofferson, 1973a, 1973b; Vorberg and Wing, 1996). There is evidence to suggest that automatic adaptation, not mediated by conscious awareness of timing perturbations, is a characteristic of phase correction, i.e., the mechanism underlying compensation for phase differences between responses and metronome. Period correction, by contrast, i.e., adjustment of the internal clock, has been suggested to depend on subjects detecting a change in timing (Repp, 2001b; Repp and Keller, in press). These two error, correction processes can be combined in formal models of sensorimotor synchronization (Mates, 1994a, 1994b; Vorberg and Wing, 1996; Vorberg and Schulze, 2002), but their respective contributions to observable behavior cannot readily be distinguished. Investigating the neurological correlates of error correction might lead to the identification of distinct neural signatures and enable more detailed insight into the coordination of these processes.

To explore the neural basis and to determine on the basis of what type of temporal information the sensorimotor system achieves the remarkably swift and precise realignment of its output to perturbations of an auditory stimulus sequence, we combined the perturbation paradigm with highdensity EEG recordings. Of primary interest were, first, auditory-evoked potentials (AEPs) elicited by the metronome stimuli and, secondly, an error-related brain potential called the error-related negativity (ERN). AEPs were used to provide information on the processing of metronome interval changes. The AEP N1 component is influenced by the repetition frequency of the eliciting stimulus, with an asymptotically increasing amplitude with decreasing stimulation frequency (Davis et al., 1966). While this is commonly attributed to refractoriness properties of auditory cortical neurons (for review, see Näätänen and Picton, 1987), Tecchio et al. (2000) found evidence that the amplitude modulation encodes changes in interval duration registered by the auditory cortex and utilized by the motor system to automatically adjust synchronization performance.

The ERN is an event-related brain potential that occurs immediately after subjects commit an error (Falkenstein et al., 1995; Gehring et al., 1995). There is evidence that it reflects activity of the anterior cingulate cortex, involved in performance monitoring, and it is sensitive to temporal aspects of response processing (Luu et al., 2000). Here, the ERN was exploited to access the processing of phase information represented in the synchronization error. A deviant metronome interval alters the phase relation between taps and metronome sequence and causes an abrupt change in tap-tone asynchrony. We anticipated that the induced change would be processed as a performance error and elicit an ERN. Given earlier findings in the oculomotor domain the generation of this component would not necessarily be

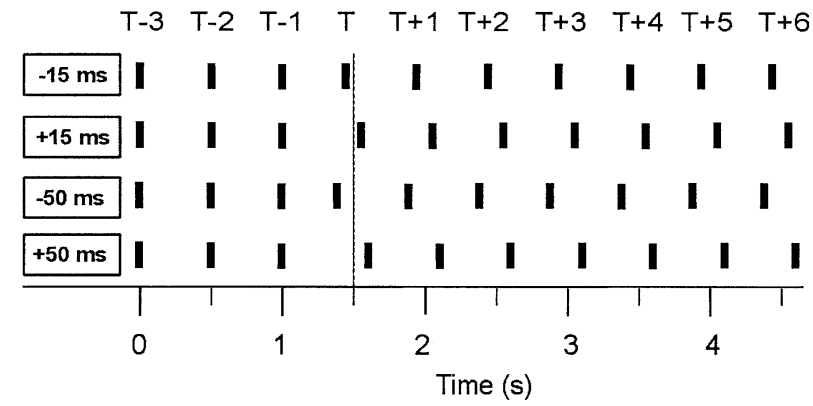

Fig. 1. Schematic representation of the stimulation conditions. The timing stimulus phase shift occurs at position $\mathrm{T}$ (time of phase shift). Both behavioral and electrophysiological data are analyzed as a function of stimulus (or tap) position, with values at T-3 to T-1 treated as baseline.

limited to situations where subjects are aware of an error (Nieuwenhuis et al., 2001).

\section{Materials and methods}

\section{Participants}

Ten subjects ( 4 men; age $25 \pm 6$ years), participated in Experiment 1. Eight participants were right handed and 2 left handed, as determined with the Edinburgh Handedness Questionnaire (Oldfield, 1971). In Experiment 2, there were 9 right-handed participants (5 men; age $26 \pm 5$ years). Data from 3 more subjects were collected but not included in the analysis because of poor signal to noise ratio. Experiment 3 had 10 right-handed participants $(6$ men; age $29 \pm 10$ years). Data from 3 other subjects were not included in the analysis because of ill-defined auditory-evoked potentials or overlap by a high-amplitude postauricular muscle reflex artifact. With the aim of reducing variability, a selection requirement in Experiment 1 was that subjects had received musical training (grade 6 or above). All subjects had normal hearing. Participants gave informed consent after explanation of the experimental procedures. The investigation was approved by the department's ethical review board.

\section{Task and procedures}

\section{Experiment 1}

Subjects were asked to tap in synchrony with an auditory stimulus sequence presented at $2 \mathrm{~Hz}$. Auditory sequences contained occasional perturbations in the form of local deviations from the standard $500 \mathrm{~ms}$ interonset interval (IOI). These perturbations (or "shifts") were of four different types ( 2 directions $\times 2$ magnitudes): increments of the standard IOI of $15 \mathrm{~ms}$, increments of $50 \mathrm{~ms}$, decrements of $15 \mathrm{~ms}$, and decrements of $50 \mathrm{~ms}$ (see Fig. 1). The 15 and $50 \mathrm{~ms}$ shift magnitudes were chosen, on the basis of relevant psychophysical work, as values likely to be below and above perception threshold respectively (Friberg and Sundberg, 1995; Repp, 2000). 
The experiment consisted of 12 experimental blocks. Within each block 10 sequences of 50 stimuli were presented, with a pause of $5 \mathrm{~s}$ between sequences. Each 50-tone sequence contained 4 shifts of the same type. Each block contained only sequences with "positive shifts" (IOI increments) or only sequences with "negative shifts" (IOI decrements). The location of the shifts within a sequence was varied, but the 4 shifts always occurred at equidistant locations (9 intervening stimuli). This block construction followed the experimental setup of Experiment 1 in Repp (2000). The experiment began with EEG electrode application and reading of the written instructions for the task. There was one practice block that included sequences for each of the four different shifts. After practice, the 12 experimental blocks were run in close succession, apart from 5-min breaks after the 4th and the 8th block. After the 12th block, the stimuli of the practice block were repeated in a passive listening condition, where subjects did not tap. Finally, subjects tapped for 3 min without auditory pacing, trying to maintain a $2-\mathrm{Hz}$ rhythm. Immediately after the experiment, subjects were debriefed with a standard set of questions.

Auditory stimuli were computer-generated $1000-\mathrm{Hz}$ pure tones of 40-ms duration presented through insert earphones at a comfortable hearing level. Tapping was performed with the index finger of the dominant hand on a low-profile force transducer mounted onto the arm rest of a comfortable arm chair. The recorded force measure was stored with the EEG data.

\section{Experiment 2}

Task and procedures were similar to those of Experiment 1 , with the exception that perturbations of the metronome sequence were always of $10 \mathrm{~ms}$ magnitude, creating deviant IOIs of 490 and $510 \mathrm{~ms}$ relative to a standard IOI of $500 \mathrm{~ms}$. Compared to 120 trials per shift in Experiment 1, we now collected 200 trials per shift.

\section{Experiment 3}

Procedures were similar to those of Experiments 1 and 2, but subjects did not tap and, therefore, did not need to attend to the auditory stimulus sequences. Thus, auditory stimuli were presented in sequences of 50 stimuli, with 10 sequences constituting 1 block. Within each sequence there were four shifts of either +50 or $-50 \mathrm{~ms}$. A total of 6 blocks was presented to each subject, yielding 120 trials per shift.

\section{EEG data acquisition and preprocessing}

EEG was recorded continuously with $\mathrm{Ag} / \mathrm{AgCl}$ electrodes from 128 scalp electrodes relative to a linked mastoids reference. The electrodes were placed according to the 10-5 extension of the International 10-20 electrode system (Oostenveld and Praamstra, 2001), using a carefully positioned nylon cap. The recording array included electrodes placed below each eye and electrodes close to the external canthus of each eye to monitor eye movements and blinks. EEG signals were amplified with a band pass of $0.16-128$ $\mathrm{Hz}$ by BioSemi Active-One amplifiers and sampled at 512 $\mathrm{Hz}$.

The continuous EEG recordings were segmented offline. The first step was a segmentation into macro-epochs comprising positions $\mathrm{T}-3$ to $\mathrm{T}+6$. These epochs were subsequently segmented in shorter epochs time-locked to the auditory stimulus at each position ( -50 to $500 \mathrm{~ms}$ ) and epochs time-locked to tap-onset at each position $\mathrm{T}-3$ to $\mathrm{T}+6$ ( -300 to $200 \mathrm{~ms}$ ). Baseline correction was performed relative to a $50 \mathrm{~ms}$ prestimulus interval and relative to the -300 to $-250 \mathrm{~ms}$, pre-tap interval, respectively. Note that taps typically precede tone onsets and that the tap at position $\mathrm{T}$ is therefore still unaffected by the perturbation. Tap onsets were identified from the force recordings using an onset detection algorithm. Trials containing artifacts were rejected before averaging using individually adjusted amplitude criteria. Eye blink artifacts were corrected using a regression approach (Gratton et al., 1983). Subsequently, the data were averaged to form ERPs, separately for each condition. For the left-handed participants, who tapped with their dominant hand, EEG channels overlying left and right hemisphere were exchanged to allow analyses together with the right-handed participants.

\section{Data analysis}

Analyses of the behavioral data focused on the tap-tone asynchronies, plotted as a function of position relative to the shift, using ANOVAs as specified under Results. Greenhouse-Geisser corrections were applied where appropriate; reported below are adjusted $P$ values.

Analogous to the analyses of the behavioral data, the AEP N1 component was quantified separately for positions T-3 through to $\mathrm{T}+6$ (see Fig. 1). N1 amplitude was measured relative to the preceding $\mathrm{P} 1$ peak at a virtual electrode created by pooling a group of 10 electrodes around the frontocentral maximum of the N1. Analyses of the ERN and the movement-preceding negative wave used a similar approach, with amplitude measures (relative to baseline) taken at a pool of 10 electrodes around the electrode with maximum amplitude.

Brain Electromagnetic Source Analysis (BESA, vs. 4.2; Scherg, 1990) was used for construction of spline interpolated isopotential maps and for EEG dipole source analysis. Dipole source analyses used a 4-shell ellipsoid head model. Analyses were performed either on grand averaged data or on data subsets, using a jackknife-based procedure (Miller et al., 1998). This involves analyses on subsamples of the grand average data set, created by iteratively leaving out one of the $N$ participants from the grand average, yielding $N$ subsets of $N-1$ participants (Leuthold and Jentzsch, 2001). Source models reported below always had a goodness of fit $>90 \%$. 

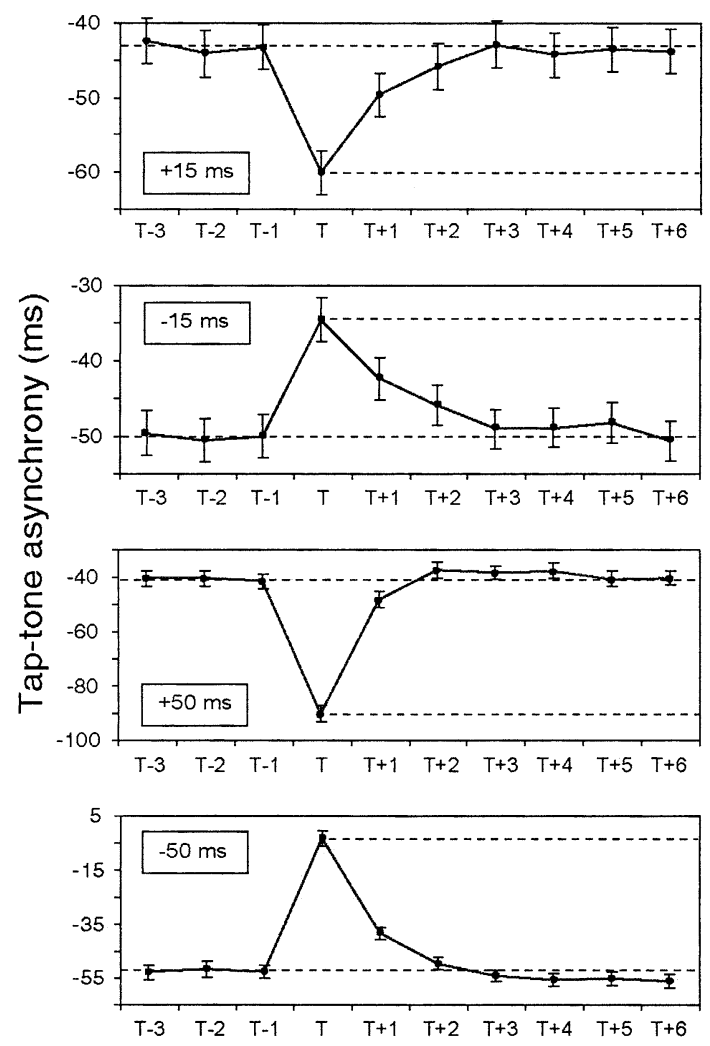

Fig. 2. Tap-tone asynchronies as a function of stimulus position. Error bars express (single) standard error. The dashed lines indicate the baseline asynchrony (mean T-3 to T-1) and the asynchrony at which tapping would continue in the absence of compensation, respectively. Note that the $y$ axes are different for 15 and $50 \mathrm{~ms}$ shifts.

\section{Results}

\section{Behavioral data}

The main analysis entered the full data set of the four different shifts in a 3-way ANOVA with within-subjects factors Magnitude (15 vs $50 \mathrm{~ms}$ ), Direction (positive vs negative), and Position (10 levels: $\mathrm{T}-3$ to $\mathrm{T}+6)$. These data are displayed in Fig. 2 as "compensation functions", i.e., tap-tone asynchronies as a function of sequence position. A pronounced change of the asynchrony naturally occurred at the shift position, and followed the direction of the shift (interaction of Direction by Position $(F(9,81)=193.7, P<$ 0.001). Moreover, there was a further 3-way interaction with Magnitude mirroring the size of the induced error at the target position $(F(9,81)=80.7, P<0.001)$.

Surprisingly, there were no significant main effects of either Magnitude $(F(1,9)<1)$ or Direction $(F(1,9)<1)$, as mean asynchronies for the four different shift types were nearly identical (between -46.1 and $-47.2 \mathrm{~ms}$ ). Related to this finding, the asynchronies during the "baseline" preceding the shifts, i.e., positions $\mathrm{T}-3$ to $\mathrm{T}-1$, differed between shift types, being smaller for positive shifts than for negative shifts (see Fig. 2). That is, the baseline was modulated in a direction opposite to that of the shift. Indeed, a separate analysis of the mean baseline asynchronies revealed a significant main effect of Direction $(F(1,9)=75.48, P<$ $0.001)$. In addition, an interaction of Magnitude and Direction $(F(1,9)=9.19, P<0.05)$ indicated that the baseline mean asynchronies for the large positive and negative amplitude shifts were further separated than the corresponding means for the small shifts. The baseline asynchrony was also used to look into serial order effects from the first to the fourth shift in the sequences. The mean baseline asynchrony showed no change from the first to fourth shift for the $15 \mathrm{~ms}$ shifts (differences $<0.5 \mathrm{~ms}$ ). By contrast, the mean baseline asynchrony drifted $\sim 5 \mathrm{~ms}$ from the first to the fourth shift for the $50 \mathrm{~ms}$ perturbations, in a direction opposite to the direction of the shift. This was reflected in a significant Magnitude by Direction by Order interaction $(F(3,27)=$ 4.62, $P<0.05)$. As further explained later, these baseline effects are probably due to overcorrection of the $50 \mathrm{~ms}$ shifts.

Fig. 2 suggests that $50 \mathrm{~ms}$ shifts were corrected faster than $15 \mathrm{~ms}$ shifts. This was addressed in an analysis of normalized values of the tap-tone asynchronies. These data are represented in Fig. 3 and suggest, furthermore, faster correction of positive shifts than negative shifts. Both features are borne out in the analysis. An ANOVA on positions $\mathrm{T}+1$ to $\mathrm{T}+6$ yielded, in addition to a significant main effect of Magnitude $(F(1,9)=17.3, P<0.01)$, significant interactions of Magnitude by Position $(F(5,45)=3.39, P<$ $0.05)$ and Direction by Position $(F(5,45)=5.81, P<0.01)$. The faster correction of the $50 \mathrm{~ms}$ shifts is probably linked to the baseline effects and overcorrection addressed in the previous paragraph and as discussed later, may reflect period correction.

Debriefings after the experiment indicated that all the participants were aware of the presence of irregularities in the metronome sequences. As the number of irregularities was underestimated, i.e., none of the participants thought that shifts had occurred in every sequence, we infer that the small shifts were difficult to perceive.

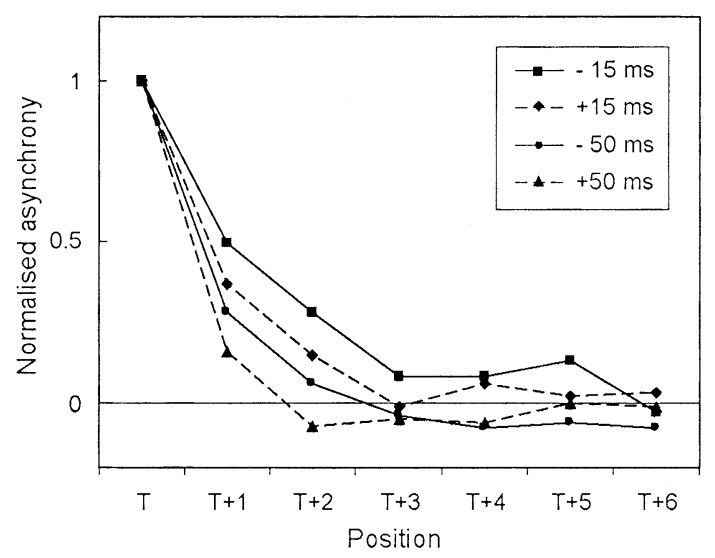

Fig. 3. Normalized relative asynchronies showing faster compensation for large relative to small magnitude shifts and for incremental relative to decremental shifts. Note overcompensation of the $50 \mathrm{~ms}$ shifts. 
$[\mathrm{A}]$

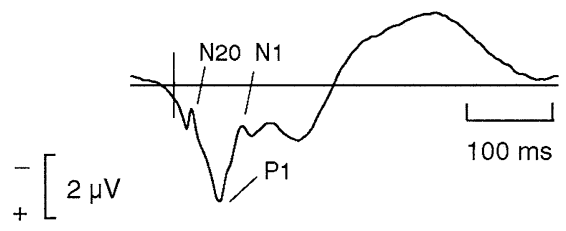

[1] Premovement negative wave

[2] Reafferent activity

[3] Postmovement positive wave

[C]

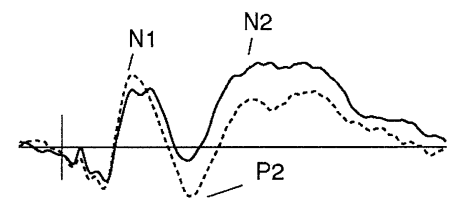

Fig. 4. Grand average ERP waveforms from location FCz, illustrating the relevant components. (A) Stimulus-locked average with labels of auditoryevoked potentials. (B) Response-locked average of the same data emphasizing the movement-related components. The waveform is aligned (relative to panel A) such that the tap precedes the stimulus by an interval equal to the mean tap-tone asynchrony. (C) Auditory-evoked potentials recorded in the passive auditory stimulation condition. Note split N1 peak in Experiment 1 (continuous line), but single peak in Experiment 2 (dotted line).

\section{Neurophysiological data: components and waveform morphology}

Fig. 4 depicts a stimulus-locked (panel A) and a response-locked (panel B) averaged waveform from a frontocentral electrode site in order to introduce the relevant components. Auditory components can be identified in the stimulus-locked waveform, beginning with the N20 and P50 (P1) components, followed by the $\mathrm{N} 1$ around $80 \mathrm{~ms}$. The relatively short peak latency of the N1 is not unusual with the stimulus presentation rate that was used (Robinson and Rudge, 1977). The N1 is followed by a second negative peak, still in the N1 latency range, around $105 \mathrm{~ms}$. As illustrated in panel $\mathrm{C}$, showing waveforms from the passive listening condition, this "splitting" of the N1 did not occur in Experiment 2. It is not considered in the following analyses.

The response-synchronized waveform is characterized by a broad premovement negative wave, followed by a negative wave of shorter duration due to reafferent activity (cf. Gerloff et al., 1998). Superimposed on the reafferent negative wave, but attenuated due to filtering, are shortlatency tactile sensory components elicited by the collision of the finger on the force plate. The reafferent negative wave is followed by a postmovement positive wave. Note that, with an average tap-tone asynchrony of approximately -45 $\mathrm{ms}$ (range -27 to $-77 \mathrm{~ms}$ ), the postmovement-positive wave tends to overlap with the auditory P1 and N1, yielding an enhancement of the P1 relative to its amplitude during passive listening. This illustrates that, unavoidably, stimu- lus-locked data still contain traces of response-related components and vice versa. This is also evident for the auditory $\mathrm{N} 2$ and the premovement negativity which are clearly superimposed in both the response-locked and the stimuluslocked data.

\section{Auditory-evoked potentials}

AEPs were analyzed in terms of the peak-to-peak P1-N1 amplitude at a frontocentral electrode pool (see materials and methods), with the P1 quantified between 49 and $51 \mathrm{~ms}$ and the N1 as the mean amplitude between 80 and $120 \mathrm{~ms}$ after stimulus onset. Analyses revealed a clear modulation of the P1-N1 amplitude as a function of tone position, with an increased amplitude following shifts that define an IOI increment $(+15$ and $+50 \mathrm{~ms})$ and a reduced amplitude following shifts defining an IOI decrement $(-15$ and -50 ms) (see Fig. 5). Note that especially for the +15 and -15 ms shifts the modulation was not confined to position $\mathrm{T}$, but lingered at subsequent positions before returning to base-
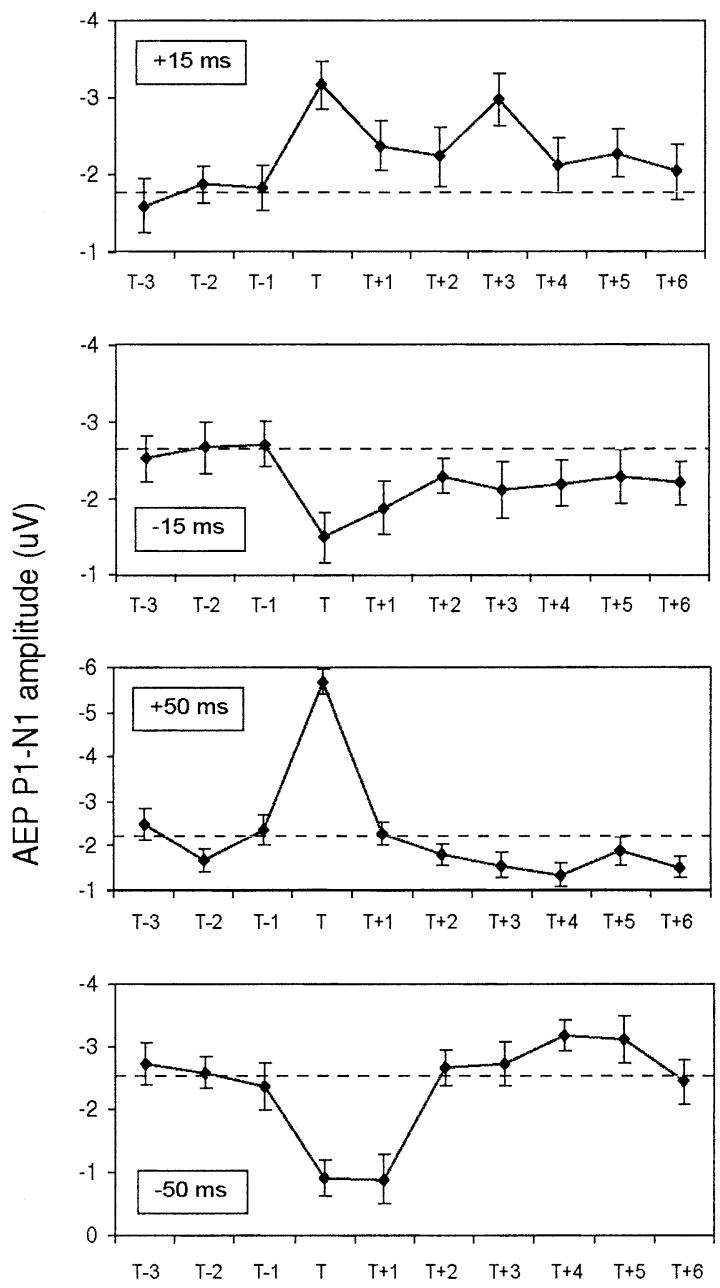

Fig. 5. AEP P1-N1 amplitude plotted as a function of position relative to the shift $(\mathrm{T})$. Error bars express standard error. Note that the $y$ axes differ between conditions. 


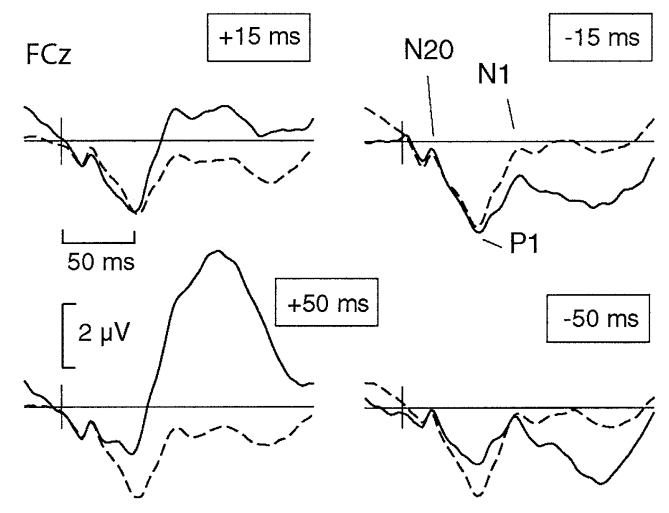

Fig. 6. AEPs at position $\mathrm{T}$ (continuous lines) superimposed on AEPs averaged across positions T-3 to T-1 (dashed lines) for each of the four different shift types. Waveforms recorded at $\mathrm{FCz}$ pooled with 4 adjacent electrodes.

line. The P1-N1 amplitude at target position was analyzed in a 2-way ANOVA across the different shift directions and magnitudes, yielding a significant main effect of Direction $(F(1,9)=49.5, P<0.001)$ and an interaction of Direction by Magnitude $(F(1,9)=20.6, P<0.01)$. The interaction was due to a stronger amplitude modulation of the AEPs with $50 \mathrm{~ms}$ shifts $(F(1,9)=58.2, P<0.001)$ than with 15 ms shifts $(F(1,9)=10.7, P<0.01)$, as tested in simple effects analyses. An alternative analysis approach comparing the $\mathrm{P} 1-\mathrm{N} 1$ amplitude at target position $\mathrm{T}$ with the mean amplitude at positions T-3 to T-1 yielded similar results (see waveforms in Fig. 6). When these latter analyses were performed with the N1 amplitude quantified in a narrow window around the peak latency $(78-82 \mathrm{~ms})$, instead of the broader window from 80 to $120 \mathrm{~ms}$ applied here, we found similar results, though the P1-N1 amplitude reduction following IOI decrements failed significance.

Despite measurement of the AEP amplitude modulation in terms of a peak-to-peak amplitude (P1-N1), the waveforms displayed in Fig. 6 raise the suspicion that the AEP modulation due to the $+50 \mathrm{~ms}$ shift is exaggerated as a result of overlap by the error-related negativity (ERN). Analyses of the ERN, reported below, confirm this suspicion.

\section{Error-related negativity}

We hypothesized that the timing perturbations, by altering the synchronization error, would elicit an error-related brain potential, best captured in response-locked averages. Fig. 7 shows the relevant data, response-synchronized to the tap at the target position. The force traces in the bottom of the figure illustrate that correction is already taking effect in the first response after the shift. The corresponding EEG traces show markedly altered waveforms for the +50 and $-50 \mathrm{~ms}$ conditions relative to a "standard" waveform averaged over positions $\mathrm{T}+6, \mathrm{~T}-3$, and $\mathrm{T}-2$. Deviations of the -50 and $+50 \mathrm{~ms}$ shift waveforms from the standard waveform are numbered 1 to 4 , and were analyzed after subtraction of the standard waveform. Difference wave 1 ( $-50 \mathrm{~ms}$ condition) could be identified, on the basis of scalp distribution and latency, as an auditory N1 response to the $-50 \mathrm{~ms}$ shifted target. Difference waves 2 and 3, peaking around $200 \mathrm{~ms}$, were considered possible ERNs and quantified in a time window from 180 to $220 \mathrm{~ms}$ at pooled frontocentral electrode sites. An ANOVA across the four shift conditions yielded a significant main effect of Magnitude $(F(1,9)=33.7, P<0.001)$, as no ERN-like waves were present for the +15 and $-15 \mathrm{~ms}$ shifts (Fig. 7, right panel). In separate $t$ tests comparing the shift waveforms against the standard waveform in the interval from 180 to $220 \mathrm{~ms}$, a significant difference was found only for the +50 ms shift $(t=-6.2, d f=1,9, P<0.001)$ and the $-50 \mathrm{~ms}$ shift $(t=-3.4, d f=1,9, P<0.01)$.

Subsequent inspection of the scalp topographies of the presumed ERNs revealed decisive differences between the topographies of difference waves 2 and 3 (see Fig. 7). The topography of wave 2 cannot be explained by a source in midline frontal structures, as required for the ERN. Instead, both the distribution and the latency fit better with an auditory $\mathrm{N} 2$ response. The topography of difference wave 3 , by contrast, can be explained by a single dipole source deep in the brain. Indeed, dipole source analysis supported a single source in medial frontal cortex, consistent with the presumed origin of the ERN in the anterior cingulate cortex (Talairach-Tournoux coordinates (mean $\pm \mathrm{SD}$ ): $X=5 \pm 2$, $Y=0 \pm 4, Z=28 \pm 3$ ).

Note that if the auditory $\mathrm{N} 1$ can be identified in the response-locked waveforms of the $-50 \mathrm{~ms}$ shift condition (wave 1 at a latency $\sim 80 \mathrm{~ms}$ ), an auditory N1 response should also be present in the waveforms of the $+50 \mathrm{~ms}$ condition, at a latency of $\sim 180 \mathrm{~ms}$. Indeed, at the appropriate latency, coinciding with the rising slope of the ERN, a scalp distribution resembling that of the auditory N1 was found, although identification of auditory sources proved impossible due to the dominant ERN.

\section{ERP correlate of period adjustment}

Following the ERN in Fig. 7, the EEG trace for the +50 ms condition shows another high-amplitude negative deflection at a latency of $\sim 400 \mathrm{~ms}$, superimposed on the late part of the movement preceding negative wave (wave 4). This negative wave had a scalp distribution similar to the ERN, with a frontocentral maximum. Dipole source analyses showed that it could be explained, like the ERN, by a single dipole in the medial frontal cortex, located $\sim 10 \mathrm{~mm}$ superior and posterior to the ERN source $(X=14 \pm 3, Y=$ $-10 \pm 4, Z=40 \pm 3$ ). As we will elaborate under Discussion, with a source location superior to the origin of the ERN, this activity might originate in the SMA and reflect the period or timekeeper adjustment also evidenced in the behavioral data. 


\section{ERP correlate of phase correction}

Analyses of the response-locked data synchronized to the tap at the shift position, illustrated in Fig. 7, indicate that they can to a large extent be explained in terms of superimposed auditory and movement-related potentials, with only for the $+50 \mathrm{~ms}$ condition an additional ERN and a possible correlate of period adjustment. However, at the latency where in the $+50 \mathrm{~ms}$ shift condition we would expect the auditory $\mathrm{N} 2$, i.e., at $\sim 280 \mathrm{~ms}$, the waveform showed a deflection of positive polarity instead of a negative deflection. By contrast, the waveform for the $-50 \mathrm{~ms}$ shift condition showed an enhancement at this latency, relative to the standard waveform. The modulation is illustrated in Fig. 7 by means of the gray area enclosed by the +50 and $-50 \mathrm{~ms}$ shift waveforms. In the +15 and $-15 \mathrm{~ms}$ shift conditions a similar modulation of the movement preceding negative wave could be observed, at the latency coinciding with the auditory N2 (Fig. 7). The amplitude modulation was quantified in a window from 250 to $300 \mathrm{~ms}$ for the $50 \mathrm{~ms}$ shifts and in a window from 275 to $325 \mathrm{~ms}$ for the $15 \mathrm{~ms}$ shifts, yielding significant amplitude differences between incremental and decremental shifts $(F(1,9)=$ $10.58, P=0.010$, and $F(1,9)=9.85, P=0.012$, respectively).

The amplitude modulation of the early phase of the movement-preceding negative wave seems not likely explained by latency variability of an overlapping, in itself invariant auditory N2. The topography of this early phase, nevertheless, resembled that of the N2 (Fig. 7, wave 5), albeit with a more central than frontocentral maximum. Source analysis identified bilateral tangential sources in suprasylvian cortex, whose activity started from about 100 ms after the tap, thus spanning the entire P1, N1, P2, and N2 sequence of AEPs. The most parsimonious explanation is therefore that these suprasylvian sources represent auditory activity that is not accurately localized to the auditory cortex due to the response-locked averaging. Alternatively, deriving support from the amplitude modulation around $300 \mathrm{~ms}$, the sources might truly reflect activity originating from the temporoparietal suprasylvian cortex, and represent activity that is related to sensorimotor integration rather than auditory activation.

\section{Experiment 2}

The behavioral results of Experiment 1 showed overcompensation of the $50 \mathrm{~ms}$ shifts. As a result, the mean tap-tone asynchrony just preceding the shifts (T-3 to T-1) drifted in a direction opposite of the direction of the shift. A smaller but likewise significant difference in the mean tap tone asynchrony from $\mathrm{T}-3$ to $\mathrm{T}-1$ was found between the $+15 \mathrm{~ms}$ and the $-15 \mathrm{~ms}$ shifts, which we attribute to a carryover of the overcompensation from the $50 \mathrm{~ms}$ shift to the $15 \mathrm{~ms}$ shift conditions. The possibility of such a carryover effect was created inadvertently by combining se- quences with positive shifts of 50 and $15 \mathrm{~ms}$ in the same experimental block (likewise for negative shifts). We also observed that the mean AEP P1-N1 amplitudes from T-3 to $\mathrm{T}-1$, i.e., preceding the shift, were nonidentical for the +15 $\mathrm{ms}$ and $-15 \mathrm{~ms}$ conditions (see Figs. 5 and 6), which emphasizes the caveat that the AEP amplitude modulation may be due to the altered temporal relation between overlapping auditory-evoked and movement-related potentials. Note further that, especially for the negative shifts, the AEP amplitude modulation appears most pronounced after rather than at the N1 peak latency (see Fig. 6). These features raise the possibility that the AEP amplitude modulation is not an $\mathrm{N} 1$ amplitude effect related to the metronome interval changes. To evaluate alternative interpretations, we conducted a second experiment with an even smaller shift magnitude $(+10$ and $-10 \mathrm{~ms})$. The use of only one shift magnitude also enabled the collection of more data per shift condition, thus improving the signal-to-noise ratio and providing better data to evaluate the nature of the AEP amplitude modulation.

\section{Results}

\section{Behavioral data}

The tap-tone asynchronies of the $+10 \mathrm{~ms}$ and the -10 ms shift conditions were entered in a 2-way ANOVA with within-subjects factor Direction (positive vs negative), and Position (10 levels: $\mathrm{T}-3$ to $\mathrm{T}+6)$. The change in the asynchrony of tone and taps, induced by the perturbations at position-T, was manifested in a significant interaction of Direction by Position $(F(9,72)=41.3, P<0.001)$. There were no significant main effects of either Direction or Position. Compensation functions constructed from the relative tap-tone asynchronies were of similar shape as the compensation functions in Experiment 1. Comparisons of normalized tap-tone asynchronies between Experiment 1 and 2 showed that $10 \mathrm{~ms}$ shifts were not compensated slower than the $15 \mathrm{~ms}$ shifts. There was no sign of overcompensation and the mean asynchronies $( \pm \mathrm{SD})$ at positions T- 3 to T- 1 were closely similar for the +10 and -10 ms shifts $(-41.5 \pm 17.1 \mathrm{~ms}$ vs $-40.7 \pm 14.1 \mathrm{~ms})$.

\section{Neurophysiological data}

Analyses of the response-locked waveforms yielded results matching those for the $15 \mathrm{~ms}$ shifts in Experiment 1 and will not be further reported. Analyses of AEPs in terms of the P1-N1 amplitude reproduced the AEP amplitude modulation seen in Experiment 1, with a modulation of the P1-N1 amplitude as a function of tone position. Similar to the results for the $15 \mathrm{~ms}$ shifts in Experiment 1, the AEP amplitude modulation was not confined to the target position, as amplitudes only returned to baseline values after several "standard" 500-ms IOI metronome periods. We will 
[A] $50 \mathrm{~ms}$ perturbations

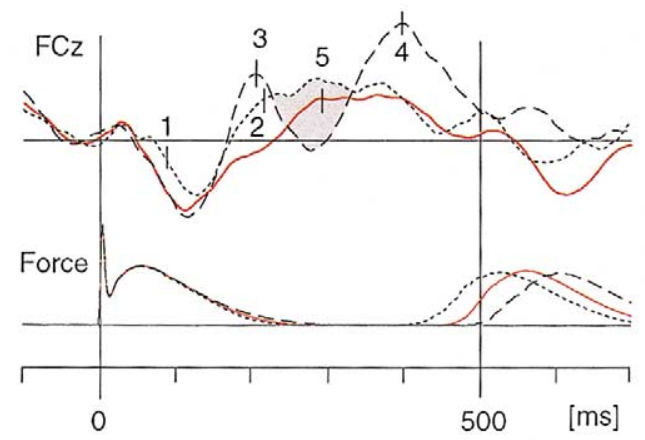

[B]

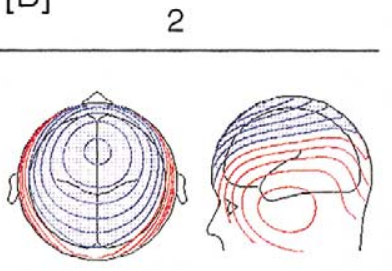

$15 \mathrm{~ms}$ perturbations

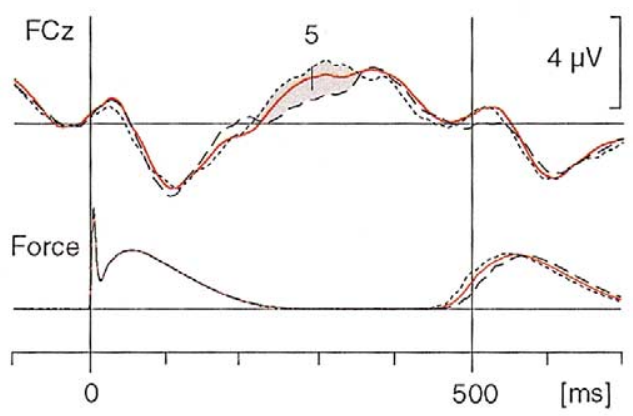

3

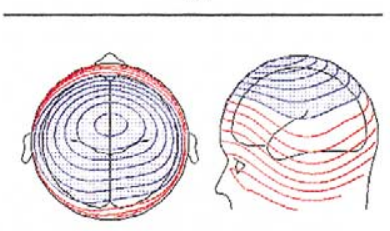

5

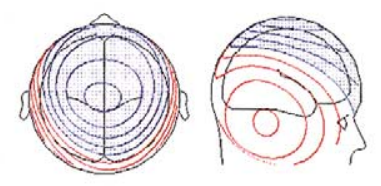

$\mathrm{FCz}$
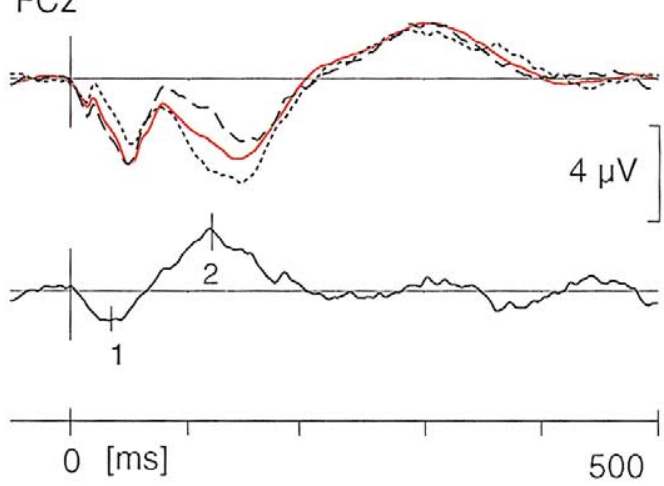

8

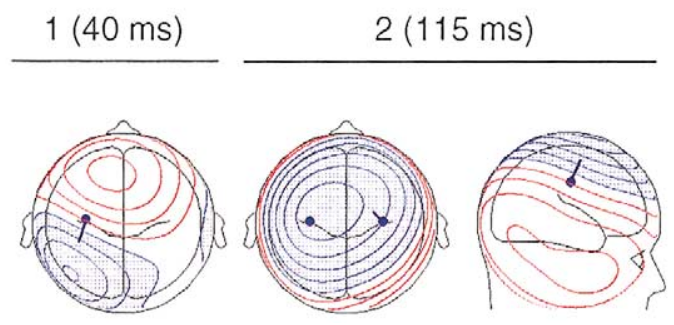

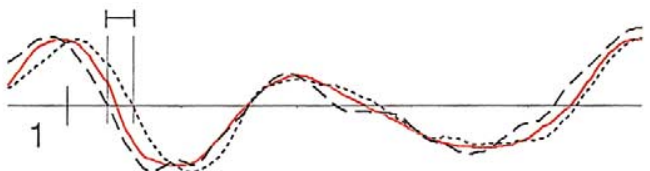

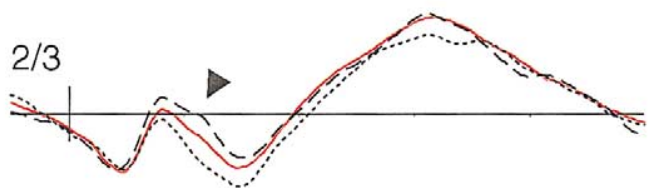

$4 / 5$

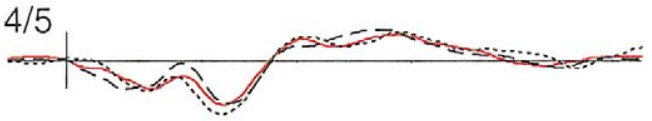

10
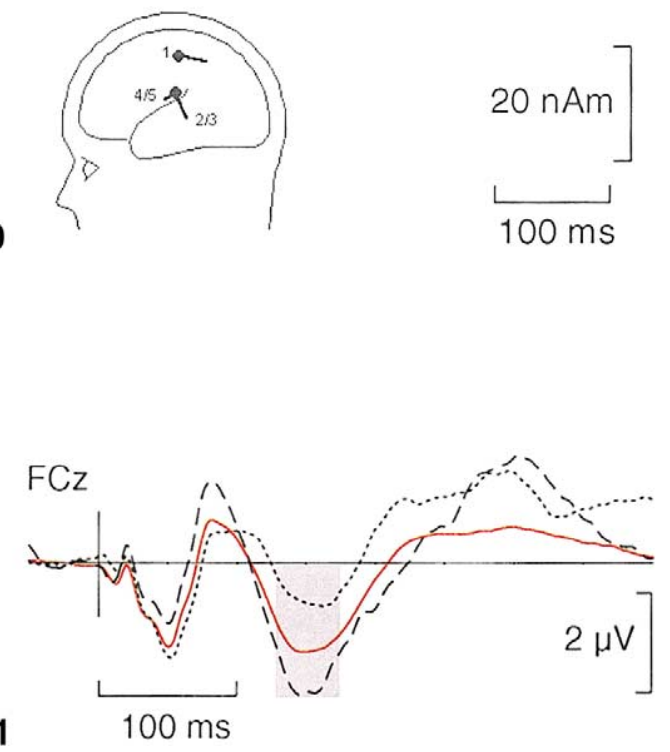
return to this issue below. The $\mathrm{P} 1-\mathrm{N} 1$ amplitude at target position was first analyzed in a 1-way ANOVA comparing the +10 and $-10 \mathrm{~ms}$ shift conditions, yielding a significant main effect of Direction $(F(1,8)=9.5, P<0.05)$. Comparing the $\mathrm{P} 1-\mathrm{N} 1$ amplitude at target position against the mean amplitude at positions T-3 to T-1 in a 2-way ANOVA yielded a significant interaction of Direction by Position $(F(1,8)=16.5, P<0.01)$. Post hoc $t$ tests confirmed an enhancement of the $\mathrm{P} 1-\mathrm{N} 1$ amplitude at the target position for the $+10 \mathrm{~ms}$ shift $(t=-3.1, d f=8, P=0.014)$ and a reduction for the $-10 \mathrm{~ms} \operatorname{shift}(t=3.7, d f=8, P=0.006)$.

Fig. 8 shows the stimulus-locked waveforms for the +10 and $-10 \mathrm{~ms}$ shifts superimposed on a baseline waveform averaged over positions T- 3 to $\mathrm{T}-1$. The difference wave, created by subtracting the response to the $-10 \mathrm{~ms}$ shift from the response to the $+10 \mathrm{~ms}$ shift, shows an early positive deflection peaking around $40 \mathrm{~ms}$ and a negative deflection peaking around $115 \mathrm{~ms}$. The positive deflection appears due to the shifted temporal relation between auditory-evoked and movement-related activity. This is illustrated by its scalp topography, which reveals underlying movement-related activity, i.e., the postmovement positive wave (see Fig. 8). The second deflection is also within the latency window of the postmovement positive wave, but more closely overlapping with its rising flank, developing into the movement-preceding negative wave. Note that the difference wave peaks markedly later than the auditory N1. This is consistent with our observation in Experiment 1 that AEP amplitude effects were most robust when evaluated in a broad window from 80 to $120 \mathrm{~ms}$, and corresponding difference waves derived for the data of Experiment $1(+15$ $\mathrm{ms}$ minus $-15 \mathrm{~ms}$ ) also peaked later than the $\mathrm{N} 1$, i.e., around $115 \mathrm{~ms}$.

The finding that the AEP amplitude modulation is not temporally coincident with the N1 requires a reconsideration of its relation to the auditory N1 component. Such a reconsideration is also encouraged by the finding that AEP

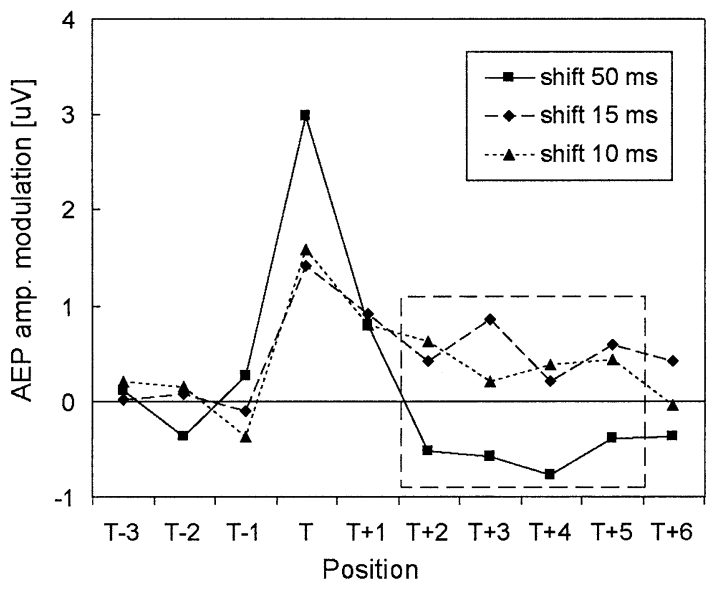

Fig. 9. Time course of the AEP amplitude modulation as a function of position relative to shift (T) for shifts of 15 and $50 \mathrm{~ms}$ (Experiment 1) and $10 \mathrm{~ms}$ (Experiment 2) magnitude. The dashed rectangle encloses positions where a significant difference between large and small shifts was found.

amplitude modulations were slow to return to the preshift baseline amplitude values. Fig. 9 plots the AEP amplitude modulation (taking absolute values and averaging positive and negative shifts) for the $10 \mathrm{~ms}$ shifts as well as the 15 and $50 \mathrm{~ms}$ shifts of Experiment 1. Note that there is a different pattern for the large $(50 \mathrm{~ms})$ and the small amplitude (10 and $15 \mathrm{~ms}$ ) shifts, with the AEP amplitude for the $50 \mathrm{~ms}$ shifts conditions dropping below the baseline value shortly after the shift, whereas the AEP amplitude for the 15 and 10 ms conditions returns to baseline very slowly. As a result, amplitude values were significantly different at positions $\mathrm{T}+2$ to $\mathrm{T}+5$ as evaluated by $t$ tests $(t=-2.4$ to $-4.4, d f$ $=27, P<0.05)$. Note that the differences in time course of the AEP amplitude modulation following large and small shifts, as illustrated in Fig. 9, have a striking resemblance to differences in the time course of the compensation functions displayed in Fig. 3. This behavior argues against the AEP

Fig. 7. (A) Response-locked data averaged with respect to the tap planned to coincide with the stimulus at T. The left panel shows the responses of the +50 $\mathrm{ms}$ condition (dashed line) and the $-50 \mathrm{~ms}$ condition (dotted line) superimposed on averaged responses from the preceding taps at T-6, T-3, and T-2 (solid line). The top traces show EEG data, the bottom traces Force. The right panel shows the same for the +15 and -15 ms conditions. The gray area marks a modulation of the initial segment of the movement-preceding negative wave. The numbers 1 and 2 refer to the displaced auditory N1 and N2 of the -50 ms condition, respectively. Numbers 3 and 4 refer to the ERN and to an ERP correlate of period adjustment in the +50 ms condition, respectively. Number 5 marks the early phase of the movement-preceding negative wave. (B) Spline-interpolated isopotential maps of difference wave 2 ( $215 \mathrm{~ms}$ ) and 3 (200 ms), and wave 5 ( $300 \mathrm{~ms})$. Scaling $0.3 \mu \mathrm{V} /$ line.

Fig. 8. Stimulus-locked traces synchronized to the $+10 \mathrm{~ms}$ shifted tone (T) (dashed line) and the $-10 \mathrm{~ms}$ shifted tone (dotted line), superimposed on the averaged responses from the three preceding stimuli from both conditions in Experiment 2 (red line). At the bottom is displayed a subtraction of the traces from the $+10 \mathrm{~ms}$ and the $-10 \mathrm{~ms}$ conditions at $\mathrm{T}$, with spline interpolated isopotential maps at the peak latencies of the positive deflection (1) and the negative deflection (2) of the subtraction waveform. The first map shows movement-related activity. The second map represents presumed auditory-somatosensory interaction.

Fig. 10. Spatiotemporal dipole source model separating movement-related positive wave from auditory activity in stimulus-locked waveforms. For ease of presentation, the activity of bilaterally symmetrical tangentially orientated sources ( 2 and 3 ) are represented in one source waveform, as are symmetrical radial sources (4 and 5). Source 1 in sensorimotor cortex accounts for the movement-related positive wave and demonstrates a latency shift of $\sim 20 \mathrm{~ms}$ for the +10 $\mathrm{ms}$ relative to the $-10 \mathrm{~ms}$ shift condition. The filled arrowhead indicates the AEP modulation, which is not eliminated by modeling of the shifted postmovement-positive wave.

Fig. 11. AEPs recorded in Experiment 3, with traces for $+50 \mathrm{~ms}$ shifts (dashed line) and $-50 \mathrm{~ms}$ shifts (dotted line) superimposed on responses following a regular $500 \mathrm{~ms}$ IOI (red line). Note the amplitude modulation in the P2 latency window, which is opposite to that illustrated in Fig. 8. The amplitude effects in the late N2 latency range are attributed to (dis)habituation. 
amplitude modulation being strictly determined by the increment or decrement of the IOI. An alternative account is that the AEP amplitude modulation is related to the tap-tone asynchrony, either in terms of varying overlap by movement-related activity, or related to the neural processing of the tap-tone asynchrony for error correction. A high correlation between the AEP modulations and the time course of the tap-tone asynchrony across $\mathrm{T}-3$ to $\mathrm{T}+6$ for each of the 10,15 , and $50 \mathrm{~ms}$ magnitude perturbations (correlations between 0.67 and 0.94 ; mean 0.83 ) raises suspicion that the modulation is indeed due to shifts in timing between auditory-evoked and movement-related activity.

\section{Dipole source analysis}

Dipole source analysis was applied on grand-averaged data and subsamples of grand averages (see materials and methods). The subtraction of the conditions with +10 and $-10 \mathrm{~ms}$ shifts was, at the peak latency of the initial positive wave $(40 \mathrm{~ms})$, modeled by a single source in sensorimotor cortex, confirming that it is due to the overlapping postmovement-positive wave (Fig. 8). The subsequent negative wave was modeled by a pair of dipole sources with a symmetry constraint on their location. These sources, fitted in an interval from 110 to $130 \mathrm{~ms}$, consistently assumed a location superior to the sources for the auditory N1 response, as identified in data from the passive listening condition, and explained the data with a residual variance always $<8 \%$ (Talairach-Tournoux coordinates $x=-1+34$ $\pm 3, y=-17 \pm 3, z=40 \pm 4$ ).

Dipole source analysis was also applied at the grand average across positions $\mathrm{T}-3$ to $\mathrm{T}-1$, subsequently using the obtained model for the waveforms at the $+10 \mathrm{~ms}$ and -10 ms shift positions (Fig. 10). This approach enabled a separation of the movement-related positive wave from auditoryevoked activity and confirmed a systematic shift in their temporal relation, induced by the timing perturbations. Thus, a model with bilateral radial and tangential sources near the supratemporal plane and a central source in the left sensorimotor cortex explained the data well (residual variance $<6 \%$ ), accounted for the temporal shift between movement-related and auditory-evoked activity, and did not eliminate the AEP modulation (Fig. 10).

Together, these analyses separate the shift-related AEP modulation from the overlapping movement-related positive wave and localize it to bilateral temporoparietal cortex. Note that these analyses do not exclude that the AEP modulation is due to varying overlap with movement preparation-related activity. This is because the tangential sources in auditory cortex can account for movement-related activity in inferior parietal cortex and even for activity generated by bilateral radial sources in the precentral gyrus, as in fact demonstrated by the good fit of the model in Fig. 10. We will take up this issue again under Discussion, where we construe the present data as evidence for a temporoparietal component contributing to the movement-preceding negative wave.

\section{Experiment 3}

The emerging possibility that AEP modulations following timing perturbations in sensorimotor synchronization are not $\mathrm{N} 1$ amplitude changes due to the varying IOI durations was further evaluated in an experiment that involved just passive listening to auditory stimulus sequences containing timing perturbations. If the above view is correct, AEPs may demonstrate $\mathrm{N} 1$ amplitude changes as a function of IOI duration, but should not exhibit the type of amplitude modulation found in Experiments 1 and 2. This could not be evaluated in the data collected during passive listening in Experiments 1 and 2. First, while the stimulus sequences did contain timing perturbations, the total duration of data collection was too short to have enough trials per perturbation type. Secondly, the passive condition always followed more than $1 \mathrm{~h}$ tapping to the same auditory stimuli, which might have affected passive auditory processing and hence the AEPs.

\section{Results}

As shown in Fig. 11, the AEP waveforms replicated the morphology found with passive auditory stimulation in Experiments 1 and 2 (see Fig. 4). Despite the suggestion of a small amplitude difference, evaluation of the N1 amplitude as a function of shift direction yielded no significant effect $(F(1,9)<1)$. In the $\mathrm{P} 2$ latency window, by contrast, there is a significant difference between the waveforms from the +50 and $-50 \mathrm{~ms}$ shift conditions, showing a resemblance with the AEP modulation found in the synchronization experiments $(F(1,9)=5.66, P<0.05)$. The resemblance is misleading, however, as the modulation is exactly opposite to that found during synchronization, with a higher P2 amplitude for the $+50 \mathrm{~ms}$ condition than the $-50 \mathrm{~ms}$ shifts. The direction of the difference entirely fits the results from previous work concerning stimulus rate effects on the amplitude of the auditory N1 component. In part of that work, namely, the N1 is measured as N1-P2 peak-to-peak amplitude (e.g., Davis et al., 1966; Butler, 1973). Indeed, evaluated in terms of N1-P2 amplitude, the difference between -50 and +50 ms shift conditions is more robust $(F(1,9)=$ $19.38, P=0.002)$. We conclude that these results replicate stimulus rate effects on the N1-P2 amplitude known in the literature. This rate effect is unambiguously different from the AEP modulation found during synchronization.

\section{General discussion}

Exploring the neurophysiology underlying rapid adjustments to timing perturbations in sensorimotor synchroniza- 
tion, this study used the ERN and AEPs to examine two sources of temporal information, that is, the tap-tone asynchrony or synchronization error and the varying interval of the auditory metronome. The presence of an ERN depended on the size and the direction of the timing perturbation. Together with the behavioral data, the ERN and the frontal midline activity immediately following it provide support for distinct phase and period error-correction mechanisms, as proposed in dual-process error-correction models (Mates, 1994a, 1994b; Repp, 2001b; Vorberg and Wing, 1996). AEP amplitude effects were observed but did not reflect metronome interval changes. As we develop below, we propose an alternative interpretation related to auditorysomatosensory interaction.

\section{Responsiveness of the ERN to synchronization error}

The ERN, signaling the activity of a neural system involved in performance monitoring (Holroyd and Coles, 2002), is not necessarily responsive to errors that are induced rather than self-made. Here, errors were induced by perturbations of the metronome sequence that, at least in the case of $50 \mathrm{~ms}$ shifts, were large enough to be perceived as irregularities in stimulation, as inferred from debriefings. That participants still generated an ERN, despite this knowledge, suggests that subjects evaluate their performance using the tap-tone asynchrony. A process evaluating the synchronization error is commonly assumed in computational models of sensorimotor synchronization (Hary and Moore, 1985; Mates, 1994a, 1994b; Pressing 1998; Schulze and Vorberg, 2002; Semjen et al, 1998; Vorberg and Wing, 1996). The ERN does not provide us with further insights into this mechanism, however, as it was only present for shifts of $+50 \mathrm{~ms}$ but not for $-50 \mathrm{~ms}$ shifts, nor for $15 \mathrm{~ms}$ shifts, either positive or negative. At first glance, the absence of an ERN for small shifts might indicate a difference between error monitoring for skeletomotor and for oculomotor control, as Nieuwenhuis et al. (2001) found an ERN to erroneous pro-saccades in an anti-saccade task, irrespective of whether subjects were aware of their errors. However, such an explanation would disregard the fundamental differences in what constitutes an error in the tasks that are compared. The absence of an ERN for small shifts is likely due to the shift-induced change of the tap-tone asynchrony being submerged by the intrinsic variability of the synchronization error.

As to the absence of an ERN for $-50 \mathrm{~ms}$ shifts, there are various possible explanations. Recall, first, that subjects tapped ahead of the metronome stimuli with a so-called negative asynchrony of $\sim 45 \mathrm{~ms}$. If, as seems to be the prevailing view (for review, see Aschersleben, 2002), this absolute negative asynchrony reflects a point of subjective synchrony, perturbations of positive and negative direction should be equally effective in triggering an error-monitoring system sensitive to the synchronization error. In absolute terms, however, positive perturbations increase the error while negative perturbations reduce it, potentially explaining our findings. Our results thus encourage a reevaluation of the concept of subjective synchrony in sensorimotor synchronization. It might be the case that the common finding of a negative asynchrony has a strategic element to it, rather than being the result of differential sensory conduction delays in the auditory and somatosensory modality (cf. Vorberg and Wing, 1996).

An equally challenging, but altogether different, explanation relates to the nature of event-related EEG components and characteristics of the corrective behavioral responses. It is debated whether averaged EEG responses reflect relatively fixed brain events coming from discrete brain regions or stimulus induced changes in the ongoing background EEG dynamics (Makeig et al., 2002; Penny et al., 2002). Under the former view, deviation from (subjective) synchrony of tap and tone in either direction might be reflected in an ERN. Under the latter view, the occurrence of an ERN is much more likely to be influenced by characteristics of the corrective behavioral response, as the corrective response affects the ongoing EEG. Different from the situation in choice response tasks, where our current knowledge of the ERN mainly stems from, corrective responses in sensorimotor synchronization do not involve deactivation and activation of different sets of effectors, but instead a temporal adjustment in the activation of the same effectors. The opposite direction of adjustments, i.e., speeding-up vs slowing down an ongoing movement, could be responsible for the different results found with -50 and $+50 \mathrm{~ms}$ shifts, given the known effects of movement-related synchronization and desynchronization in the alpha frequency band (Pfurtscheller and Lopes da Silva, 1999; Salmelin et al., 1995). These effects mainly refer to sensorimotor areas and alpha rhythms, but midline frontal areas presumed to generate the ERN might demonstrate similar behavior in the theta band (cf. Luu and Tucker, 2001). Results indicating that error correction starts well before the appearance of the ERN lend further support to this explanation (Rodriguez-Fornells et al., 2002)

The finding that an ERN was elicited by at least one type of perturbation used in this investigation speaks to an important issue regarding the nature of the ERN. Whereas the ERN was originally suggested to reflect the operation of an error-detection mechanism (Falkenstein et al., 1995; Gehring et al., 1995), it was recently proposed to reflect evaluative processes sensitive to response conflict (e.g., Carter et al., 1998; Gehring and Fencsik, 2001). This proposal entails that the ERN signals conditions where errors are likely to occur rather than errors themselves. Contrary to this view, our findings demonstrate that response conflict is not a necessary element of the conditions determining the generation of an ERN. 
Neural correlates of period correction: medial premotor cortex activation

Correction of the large $50 \mathrm{~ms}$ stimulus phase shifts proceeded faster than the small magnitude perturbations and involved some degree of overcompensation. Related phenomena have been observed before and have been attributed to the engagement of period correction mechanisms in addition to the phase correction that one expects to be active (cf. Repp, 2001a). According to computational models of sensorimotor synchronization, phase corrections are implemented by adjusting the temporal placement of each tap by some proportion of the synchronization error associated with the preceding tap (Hary and Moore, 1985; Mates, 1994a, 1994b; Vorberg and Wing, 1996), without adjustment of the timekeeper period. While such an adjustment is not required with stimulus phase shifts, it appears that large size shifts invoked additional period correction, possibly mediated by an awareness of the timing perturbation (cf. Repp, 2001b). This interpretation is supported by qualitative differences between the movement-related potentials recorded for 50 and $15 \mathrm{~ms}$ perturbations at the position just following the shift (see Fig. 7). The terminal phase of the movement-preceding negativity of the $+50 \mathrm{~ms}$ condition demonstrated a marked enhancement explained by frontal midline activity. This activity originated from a location more superior than the ERN source in the anterior cingulate cortex, corresponding with the supplementary motor area (SMA). SMA involvement (as well as anterior cingulate cortex activation) has been found in imaging studies of sensorimotor synchronization/continuation (Jäncke et al., 2000; Rao et al., 1997; Stephan et al., 2002) or temporal processing (Macar et al., 2002; Schubotz and Von Cramon, 2001). Especially relevant here, Rao et al. (1997) found SMA activation with continuation but not with synchronization, while Stephan et al. (2002) observed SMA activation when subjects were aware of perturbations. These observations fit with a role of the SMA in explicit timing that is called upon when timekeeper interval adjustments are required. Just as with the ERN, a question arises again as to why the SMA activity related to period corrections is only found in the $+50 \mathrm{~ms}$ condition and not with $-50 \mathrm{~ms}$ shifts. We surmise that the SMA is active in both conditions, but that its expression in the scalp-recorded EEG is facilitated in the $+50 \mathrm{~ms}$ and suppressed in the $-50 \mathrm{~ms}$ condition due to movement-related synchronization and desynchronization effects in lower frequency bands.

\section{AEPs and auditory-somatosensory interaction}

The ERN findings indicate that the tap-tone asynchrony is evaluated at a conscious level, but cannot be taken as evidence that the synchronization error represents temporal information that is actually used for sensorimotor synchronization. Formal models of sensorimotor synchronization commonly attribute a primary role to the trial-to-trial eval- uation of the synchronization error (Hary and Moore, 1985; Mates, 1994a, 1994b; Pressing, 1998; Semjen et al., 1998; Vorberg and Wing, 1996). Models based on period information are also conceivable, however (Michon, 1967; Thaut et al., 1998a, 1998b). Tecchio et al. (2000) claimed that motor adjustments to step changes in a metronome sequence are guided by the auditory system detecting metronome period change, as evidenced by an amplitude modulation of the magnetoencephalographic (MEG) analogue of the N1. In our data, compelling evidence for rejecting the AEP modulation being due to period change comes from Experiment 3 with passive auditory stimulation, where we found that IOI increments yield higher N1-P2 amplitudes than IOI decrements, in agreement with existing evidence from research on auditory refractoriness effects on the N1 (e.g., Davis et al., 1966; Butler, 1973). During tapping, however, the same IOI changes were accompanied by amplitude modulations in the N1-P2 latency range of opposite direction (see Fig. 8 and 11), arguing against an explanation of the latter effect as determined by the metronome interval. Tecchio et al. (2000) were not in a position to find this modulation, as they only recorded MEG during passive listening.

A further argument against the AEP modulation reflecting metronome interval change is that it covaried with the tap-tone asynchrony at positions other than $\mathrm{T}$, ie., positions where the metronome interval was the standard $500 \mathrm{~ms}$. This suggests that it is probably not "shift-induced," but instead reflects a varying overlap of stimulus-locked AEPs by activity that is more closely time-locked to movement. This feature does not render the modulation irrelevant to sensorimotor synchronization. Rather, it implies that the modulation reveals activity that is not only present at shift positions, but is instead a regular component of the brain electrical activity during sensorimotor synchronization. We hypothesize that the AEP modulation represents auditorysomatosensory activity supporting sensorimotor coordination. The phase shift of auditory and movement-related activity helped to reveal this activity, and the subtraction of AEPs due to incremental and decremental shifts, which subtracts out phase-locked auditory activity, gives probably the most reliable estimate of its origin. Nevertheless, the corresponding latency window of the response-locked data (150-200 ms) was also explained by bilateral tangential sources in suprasylvian cortex, their activity peaking around $300 \mathrm{~ms}$. It is conceivable that this interval, which spans the auditory N1, P2, and N2 components, as well as the initial phase of the movement-preceding negativity, does not just consist of superimposed auditory and movement-related potentials, but also comprises activity that couples the two. Recall that the shift-related modulation around $300 \mathrm{~ms}$, illustrated for Experiment 1 in Fig. 7, but similarly present in Experiment 2, was difficult to explain on the basis of superposition, supporting the possibility of activity related to sensorimotor coupling. Such activity might be regarded as embodying an "attentional oscillator" (Large and Jones, 1995; Repp, 2001a) that monitors the stimulus sequence and 


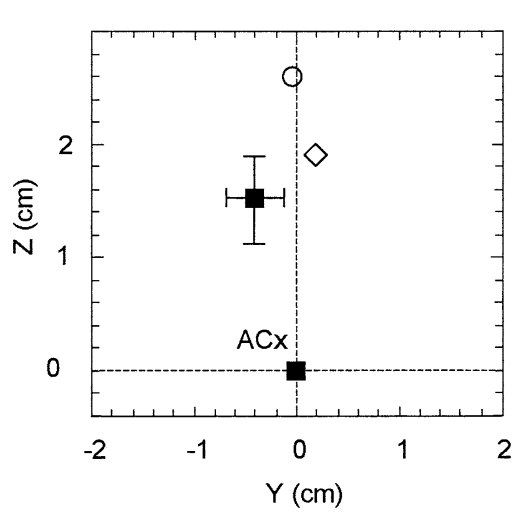

Fig. 12. Graph modified from Lütkenhöner et al. (2002) with the mean location of their SII sources (open circle) and auditory-somatosensory interaction fields (open diamond), as identified with MEG. The location of the sources explaining the AEP modulation in the present study (Experiment 2), relative to the auditory cortex (ACx), is indicated by the closed square with (1 SD) error bars.

registers (subliminal) deviations from temporal expectations to inform the motor system and implement phase corrections.

The distribution of the shift-related activity in our study, and the modeling results, are compatible with bilateral auditory-somatosensory activity in secondary somatosensory cortex SII (see Fig. 12), in line with EEG and MEG work on auditory-somatosensory integration using passive bimodal sensory stimulation (Foxe et al., 2000; Greenwood and Goff, 1987; Huttunen et al., 1987; Lütkenhöner et al., 2002; Okajima et al., 1995). Particularly relevant here, Huttunen et al. (1987) reported auditory-somatosensory interaction effects manifested in an amplitude reduction of the AEP P2 deflection. A more recent MEG study found auditory-somatosensory interaction fields, isolated by subtracting the responses to simultaneous presentation of auditory and tactile stimuli from the summed responses to separate presentations of the two types of stimuli (Lütkenhöner et al., 2002). The interaction fields were bilateral in some of the participants and were attributed to SII, although the authors acknowledge significant interindividual variability. Apart from SII, auditory cortical structures are also possible generator sites of auditory-somatosensory interaction activity. In monkey auditory association cortex, Schroeder et al. (2001) identified a site of areal convergence responsive to both auditory and somatosensory stimuli. A possible human homologue of this area $\mathrm{CM}$ was subsequently identified with fMRI (Foxe et al., 2002).

As to the functional significance of auditory-somatosensory interaction, authors have speculated on a role in spatial localization, accommodated within a division of the auditory system in "what" and "where" pathways, as proposed by Rauschecker and Tian (2000), in analogy to the concept of ventral and dorsal visual processing streams. Likewise, Repp (2000, 2001a) associated the compensation of subliminal timing perturbations in tapping with an auditory analogue of the dorsal visual stream, conceived more broadly as an auditory processing stream for the control of action (cf. Milner and Goodale, 1995). Repp and Penel (2002) observed a stronger reliance on auditory than on visual metronome stimuli in automatic motor adjustments for stimulus phase shifts, attesting to an auditory dominance in motor timing and privileged access of auditory temporal information to the motor system. Based on such evidence, integration of auditory and somatosensory information for the temporal control of movement, whether it takes place in auditory association cortex (Schroeder et al., 2001; Foxe et al., 2002) or in SII (Lütkenhöner et al., 2002), might well be a functional component of such a broader defined dorsal auditory stream that is not exclusively linked to auditory spatial processing but dedicated to the online control of action.

\section{Relation to previous work}

Kelso and co-workers studied EEG and MEG correlates of sensorimotor synchronization during transitions between different coordination modes (syncopation to synchronization) (Fuchs et al., 2000; Mayville et al., 2001). Such transitions were induced by increasing the metronome rate and typically occurred around $2 \mathrm{~Hz}$. The authors report a steep decrement of auditory response amplitudes around this stimulation frequency, which they regard as leading in to a large-scale reorganization of cortical activity, accompanying the behavioral transition in sensorimotor coordination. This contrasts sharply with our AEP data. Our AEPs recorded during tapping were of low amplitude, but source analysis still allowed the identification of both the tangential and radial source components of the N1 (cf. Scherg et al., 1989). The auditory response decrement suggested by Kelso and co-workers is also steeper than previously reported in work on refractoriness properties of the auditory N1 (Näatänen and Picton, 1987; Budd et al., 1998) and may be due to the modeling approach using an auditory control condition not matched to the stimulus rates during tapping. Although the present study did not address transitions between coordination modes, our data can to a large degree be explained on the basis of superimposed auditory and movement-related activity while interactions between the auditory and the sensorimotor system do not seem intractable.

Müller et al. (2000), in another MEG investigation, identified inferior $\mathrm{S} 1$ as possibly involved in sensorimotor synchronization, suggesting that it has a role in evaluating the temporal match of sensorimotor and auditory events. Given the variability of audiotactile responses (cf. Lütkenhöner et al., 2002), this activity could represent the same type of auditory-somatosensory interaction as Lütkenhöner et al. localized to SII, thereby supporting the interpretation that we proposed of our data. Relevant here, activation of SII has also been confirmed in functional imaging of sensorimotor synchronization (Mima et al., 1999), albeit not in all the relevant studies (e.g., Jäncke et al., 2000; Rao et al., 1997). 


\section{Conclusion}

As a result of using both large and small timing perturbations (stimulus phase shifts) in auditory stimulus sequences guiding sensorimotor synchronization, the present study not only activated the expected phase-correction mechanisms but also invoked period-correction mechanisms. One of the main results is that these mechanisms can be distinguished neurophysiologically. The former seem to be mediated by auditory-somatosensory activity probably generated in SII. The latter is represented by frontal midline activation that we localized to the SMA. This separation provides support for two-level timing models that incorporate a central timer and a sensorimotor level accommodating phase errors (Vorberg and Wing, 1996). Although the results broadly support this two-level model, they do not yet allow very detailed insight in the cortical mechanisms supporting auditory-motor interactions. Further research is needed both regarding the precise mechanisms underlying the evaluation of the synchronization error and regarding the implementation of corrective action.

\section{Acknowledgments}

We thank Nick Roach and Ellen Seiss for technical and programming support. Bruno Repp provided very helpful comments on an earlier poster presentation of these data. The research was supported by a grant from the MRC.

\section{References}

Aschersleben, G., 2002. Temporal control of movements in sensorimotor synchronization. Brain Cogn. 48, 66-79.

Budd, T.W., Barry, R.J., Gordon, E., Rennie, C., Michie, P.T., 1998. Decrement of the N1 auditory event-related potential with stimulus repetition: habituation vs. refractoriness. Int. J. Psychophysiol. 31, 51-68.

Butler, R.A., 1973. The cumulative effects of different stimulus repetition rates on the auditory evoked response in man. Electroencephalogr. Clin. Neurophysiol. 35, 337-45.

Carter, C.S., Braver, T.S., Barch, D.M., Botvinick, M.M., Noll, D., Cohen, J.D., 1998. Anterior cingulate cortex, error detection, and the online monitoring of performance. Science 280, 747-9.

Davis, H., Mast, T., Yoshie, N., Zerlin, S., 1966. The slow response of the human cortex to auditory stimuli: recovery process. Electroencephalogr. Clin. Neurophysiol. 21, 105-13.

Falkenstein, M., Hohnsbein, J., Hoormann, J., 1995. Event-related potential correlates of errors in reaction tasks. Electroencephalogr. Clin. Neurophysiol. Suppl. 44, 287-96.

Foxe, J.J., Morocz, I.A., Murray, M.M., Higgins, B.A., Javitt, D.C., Schroeder, C.E., 2000. Multisensory auditory-somatosensory interactions in early cortical processing revealed by high-density electrical mapping. Brain Res. Cogn. Brain Res. 10, 77-83.

Foxe, J.J., Wylie, G.R., Martinez, A., Schroeder, C.E., Javitt, D.C., Guilfoyle, D., Ritter, W., Murray, M.M., 2002. Auditory-somatosensory multisensory processing in auditory association cortex: an fMRI study. J. Neurophysiol. 88, 540-3.

Friberg, A., Sundberg, J., 1995. Time discrimination in a monotonic, isochronous sequence. J. Acoust. Soc. Am. 98, 2524-2531.
Fuchs, A., Mayville, J.M., Cheyne, D., Weinberg, H., Deecke, L., Kelso, J.A., 2000. Spatiotemporal analysis of neuromagnetic events underlying the emergence of coordinative instabilities. NeuroImage 12, 71-84.

Gehring, W.J., Coles, M.G., Meyer, D.E., Donchin, E., 1995. A brain potential manifestation of error-related processing. Electroencephalogr. Clin. Neurophysiol. Suppl. 44, 261-72.

Gehring, W.J., Fencsik, D.E., 2001. Functions of the medial frontal cortex in the processing of conflict and errors. J. Neurosci. 21, 9430-7.

Gerloff, C., Uenishi, N., Hallett, M., 1998. Cortical activation during fast repetitive finger movements in humans: dipole sources of steady-state movement-related cortical potentials. J. Clin. Neurophysiol. 15, 502513.

Gratton, G., Coles, M.G.H., Donchin, E., 1983. A new method for off-line removal of ocular artifact. Electroenceph. Clin. Neurophysiol. 55, 468484.

Greenwood, P.M., Goff, W.R., 1987. Modification of median nerve somatic evoked potentials by prior median nerve, peroneal nerve, and auditory stimulation. Electroencephalogr. Clin. Neurophysiol. 68, 295302.

Harrington, D.L., Haaland, K.Y., Knight, R.T., 1998. Cortical networks underlying mechanisms of time perception. J. Neurosci. 18, 1085-95.

Hary, D., Moore, G.P., 1985. Temporal tracking and synchronization strategies. Hum. Neurobiol. 4, 73-9.

Holroyd, C.B., Coles, M.G., 2002. The neural basis of human error processing: reinforcement learning, dopamine, and the error-related negativity. Psychol. Rev. 109, 679-709.

Huttunen, J., Hari, R., Vanni, S., 1987. Crossmodal interaction is reflected in vertex potentials but not in evoked magnetic fields. Acta Neurol. Scand. 75, 410-6.

Jäncke, L., Loose, R., Lutz, K., Specht, K., Shah, N.J., 2000. Cortical activations during paced finger-tapping applying visual and auditory pacing stimuli. Brain Res. Cogn. Brain Res. 10, 51-66.

Large, E.W., Jones, M.R., 1999. The dynamics of attending: how people track time-varying events. Psychol Rev. 106, 119-159.

Leuthold, H., Jentzsch, I., 2001. Neural correlates of advance movement preparation: a dipole source analysis approach. Brain Res. Cogn. Brain Res. 12, 207-24.

Lütkenhöner, B., Lammertmann, C., Simoes, C., Hari, R., 2002. Magnetoencephalographic correlates of audiotactile interaction. NeuroImage $15,509-22$.

Luu, P., Flaisch, T., Tucker, D.M., 2000. Medial frontal cortex in action monitoring. J. Neurosci. 20, 464-9.

Luu, P., Tucker, D.M., 2001. Regulating action: alternating activation of midline frontal and motor cortical networks. Clin. Neurophysiol. 112, 1295-306.

Macar, F., Lejeune, H., Bonnet, M., Ferrara, A., Pouthas, V., Vidal, F., Maquet, P., 2002. Activation of the supplementary motor area and of attentional networks during temporal processing. Exp. Brain Res. 142, 475-85.

Makeig, S., Westerfield, M., Jung, T.P., Enghoff, S., Townsend, J., Courchesne, E., Sejnowski, T.J., 2002. Dynamic brain sources of visual evoked responses. Science 295, 690-4.

Mates, J., 1994a. A model of synchronization of motor acts to a stimulus sequence. I. Timing and error corrections. Biol. Cybern. 70, 463-73.

Mates, J., 1994b. A model of synchronization of motor acts to a stimulus sequence. II. Stability analysis, error estimation and simulations. Biol. Cybern. 70, 475-84.

Mayville, J.M., Fuchs, A., Ding, M., Cheyne, D., Deecke, L., Kelso, J.A., 2001. Event-related changes in neuromagnetic activity associated with syncopation and synchronization timing tasks. Hum. Brain Mapp. 14, 65-80.

Michon, J.A., 1967. Timing in Temporal Tracking. Van Gorcum, Assen, NL.

Miller, J., Patterson, T., Ulrich, R., 1998. Jackknife-based method for measuring LRP onset latency differences. Psychophysiology 35, 99 115 . 
Milner, A.D., Goodale, M.A., 1995. The Visual Brain in Action. OUP, Oxford.

Mima, T., Sadato, N., Yazawa, S., Hanakawa, T., Fukuyama, H., Yonekura, Y., Shibasaki, H., 1999. Brain structures related to active and passive finger movements in man. Brain 122, 1989-97.

Müller, K., Schmitz, F., Schnitzler, A., Freund, H.J., Aschersleben, G., Prinz, W., 2000. Neuromagnetic correlates of sensorimotor synchronization. J. Cogn. Neurosci. 12, 546-55.

Näätänen, R., Picton, T., 1987. The N1 wave of the human electric and magnetic response to sound: a review and an analysis of the component structure. Psychophysiology 24, 375-425.

Nieuwenhuis, S., Ridderinkhof, K.R., Blom, J., Band, G.P., Kok, A., 2001. Error-related brain potentials are differentially related to awareness of response errors: evidence from an antisaccade task. Psychophysiology $38,752-60$.

Okajima, Y., Chino, N., Takahashi, M., Kimura, A., 1995. Effects of visual and auditory stimuli on median nerve somatosensory evoked potentials in man. Electromyogr. Clin. Neurophysiol. 35, 251-6.

Oldfield, R.C., 1971. The assessment and analysis of handedness: the Edinburgh inventory. Neuropsychologica 9, 97-113.

Oostenveld, R., Praamstra, P., 2001. The five percent electrode system for high-resolution EEG and ERP measurements. Clin. Neurophysiol. 112, 713-19.

Penny, W.D., Kiebel, S.J., Kilner, J.M., Rugg, M.D., 2002. Event-related brain dynamics. Trends Neurosci. 25, 387-9.

Pfurtscheller, G., Lopes da Silva, F.H., 1999. Event-related EEG/MEG synchronization and desynchronization: basic principles. Clin. Neurophysiol. 110, 1842-57.

Pressing, J., 1998. Error correction processes in temporal pattern production. J. Math. Psychol. 42, 63-101.

Rao, S.M., Harrington, D.L., Haaland, K.Y., Bobholz, J.A., Cox, R.W., Binder, J.R., 1997. Distributed neural systems underlying the timing of movements. J. Neurosci. 17, 5528-35.

Rauschecker, J.P., Tian, B., 2000. Mechanisms and streams for processing of "what" and "where" in auditory cortex. Proc. Natl. Acad. Sci. USA 97, 11800-6.

Repp, B.H., 2000. Compensation for subliminal timing perturbations in perceptual-motor synchronization. Psychol. Res. 63, 106-28.

Repp, B.H., 2001a. Phase correction, phase resetting, and phase shifts after subliminal timing perturbations in sensorimotor synchronization. J. Exp. Psychol. Hum. Percept. Perform. 27, 600-21.

Repp, B.H., 2001b. Processes underlying adaptation to tempo changes in sensorimotor synchronization. Hum. Mov. Sci. 20, 277-312.

Repp, B.H., Keller, P.E., In press. Adaptation to tempo changes in sensorimotor synchronization: effects of intention, attention, and awareness. Q. J. Exp. Psychol.

Repp, B.H., Penel, A., 2002. Auditory dominance in temporal processing: new evidence from synchronization with simultaneous visual and auditory sequences. J. Exp. Psychol. Hum. Percept. Perform. 28, 108599.

Robinson, K., Rudge, P., 1977. Abnormalities of the auditory evoked potentials in patients with multiple sclerosis. Brain 100, 19-40.
Rodriguez-Fornells, A., Kurzbuch, A.R., Münte, T.F., 2002. Time course of error detection and correction in humans: neurophysiological evidence. J. Neurosci. 22, 9990-6.

Salmelin, R., Hamalainen, M., Kajola, M., Hari, R., 1995. Functional segregation of movement-related rhythmic activity in the human brain. NeuroImage 2, 237-43.

Scherg, M., 1990. Fundamentals of dipole source potential analysis, in: Grandori, F., Hoke, M., Romani, G.L. (Eds.), Auditory Evoked Magnetic Fields and Electric Potentials. Advances in Audiology, Vol. 6. Karger, Basel, pp. 40-69.

Scherg, M., Vajsar, J., Picton, T.W., 1989. A source analysis of the late human auditory evoked potentials. J. Cogn. Neurosci. 1, 336-355.

Schroeder, C.E., Foxe, J.J., 2002. The timing and laminar profile of converging inputs to multisensory areas of the macaque neocortex. Brain Res. Cogn. Brain Res. 14, 187-98.

Schroeder, C.E., Lindsley, R.W., Specht, C., Marcovici, A., Smiley, J.F., Javitt, D.C., 2001. Somatosensory input to auditory association cortex in the macaque monkey. J. Neurophysiol. 85, 1322-7.

Schubotz, R.I., von Cramon, D.Y., 2001. Interval and ordinal properties of sequences are associated with distinct premotor areas. Cereb. Cortex $11,210-22$

Schulze, H.H., Vorberg, D., 2002. Linear phase correction models for synchronization: parameter identification and estimation of parameters. Brain Cogn. 48, 80-97.

Semjen, A., Vorberg, D., Schulze, H., 1998. Getting synchronized with the metronome: comparisons between phase and period correction. Psychol. Res. 61, 44-55

Stephan, K.M., Thaut, M.H., Wunderlich, G., Schicks, W., Tian, B., Tellmann, L., Schmitz, T., Herzog, H., McIntosh, G.C., Seitz, R.J., Homberg, V., 2002. Conscious and subconscious sensorimotor synchronization: prefrontal cortex and the influence of awareness. NeuroImage 15, 345-52.

Tecchio, F., Salustri, C., Thaut, M.H., Pasqualetti, P., Rossini, P.M., 2000. Conscious and preconscious adaptation to rhythmic auditory stimuli: a magneto-encephalographic study of human brain responses. Exp. Brain Res. 135, 222-30.

Thaut, M.H., Miller, R.A., Schauer, L.M., 1998a. Multiple synchronization strategies in rhythmic sensorimotor tasks: phase vs. period correction. Biol. Cybern. 79, 241-50.

Thaut, M.H., Tian, B., Azimi-Sadjadi, M.R., 1998b. Rhythmic finger tapping to cosine wave modulated metronome sequences: evidence of subliminal entrainment. Hum. Mov. Sci. 17, 839-863.

Vorberg, D., Wing, A., 1996. Modeling variability and dependence in timing, in: Heuer, H., Keele, S.W. (Eds.), Handbook of Perception and Action: Motor skills Vol. 3. Academic Press, London, pp. 181-262.

Vorberg, D., Schulze, H.H., 2002. Linear phase-correction in synchronization: predictions, parameter estimation, and simulations. J. Math. Psychol. 46, 56-87.

Wing, A.M., Kristofferson, A.B., 1973a. The timing of interresponse intervals. Percept. Psychophys. 13, 455-460.

Wing, A.M., Kristofferson, A.B., 1973b. Response delays and the timing of discrete motor responses. Percept. Psychophys. 14, 5-12. 